\title{
Facet-Selective Dissociation and Radical-Mediated Reaction of
}

\section{Dibenzotetrathiafulvalene Molecules on Low-Index Copper Surfaces}

\author{
Jun Zhang ${ }^{1,2,3, \dagger}$, Linwei Zhou ${ }^{1, \dagger}$, Pengcheng Chen $^{2}$, Bingkai Yuan ${ }^{2}$, Zhihai
}

$$
\text { Cheng }{ }^{1,2, *} \text {, Wei Ji }{ }^{1, *} \text { and Xiaohui Qiu }{ }^{2,4 *}
$$

${ }^{1}$ Beijing Key Laboratory of Optoelectronic Functional Materials \& Micro-nano Devices, Department of Physics, Renmin University of China, Beijing 100872, China ${ }^{2}$ CAS Key Laboratory of Standardization and Measurement for Nanotechnology, CAS Center for Excellence in Nanoscience, National Center for Nanoscience and Technology, Beijing 100190, China

${ }^{3}$ Key Laboratory of Polar Materials and Devices Ministry of Education, Department of Optoelectronics, East China Normal University, Shanghai 200241, China

${ }^{4}$ University of Chinese Academy of Sciences, Beijing 100049, China

$\dagger$ J.Z. and L.Z. contributed equally to this work.

* Electronic address: zhihaicheng@ruc.edu.cn,wji@ruc.edu.cn, xhqiu@nanoctr.cn

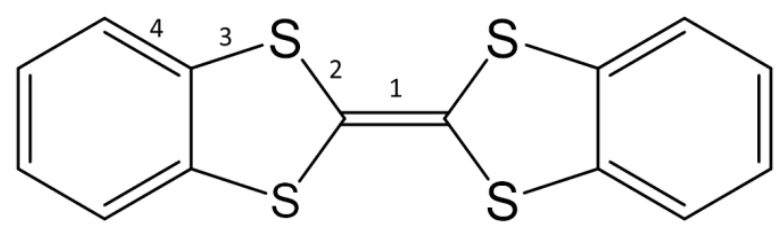

Figure S1: Chemical structure of the DBTTF molecule, in which the four specific chemical bonds are highlighted: $\mathrm{C}_{\mathrm{Et}}-\mathrm{C}_{\mathrm{Et}}$ (Bond 1), $\mathrm{C}_{\mathrm{Et}}-\mathrm{S}$ (Bond 2), $\mathrm{C}_{\mathrm{Ph}}-\mathrm{S}$ (Bond 3) and $\mathrm{C}_{\mathrm{Ph}}-\mathrm{C}_{\mathrm{Ph}}$ (Bond 4) bonds. Et is the abbreviation of ethylene. $\mathrm{Ph}$ is the abbreviation of phenyl. After adsorption on the substrates, the $\mathrm{C}_{\mathrm{Et}}-\mathrm{S}$ and $\mathrm{C}_{\mathrm{Ph}}-\mathrm{S}$ bonds are weakened and elongated due to the chemical interactions between the $\mathrm{S}$ atoms and substrate copper atoms and could be further broken due to thermal activation at different substrate temperatures. 


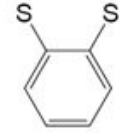

1,2-benzenedithiolate

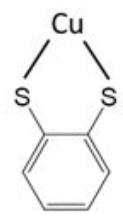

1,2-benzenedithiolate-Cu Complex

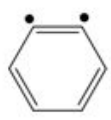

Phenyl diradical

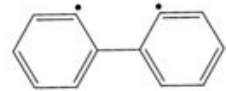

Biphenyl diradical

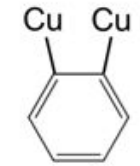

Phenyl-Cu(110)

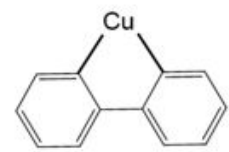

Biphenyl-Cu Complex

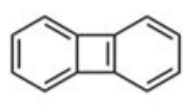

Biphenylene

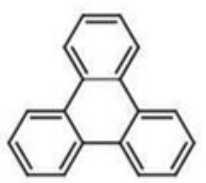

Triphenylene

Figure S2: Summary of the synthesized chemical species for DBTTF molecules on $\mathrm{Cu}(110)$ and $\mathrm{Cu}(100)$. Their names used in this paper are also supplied for clarity.

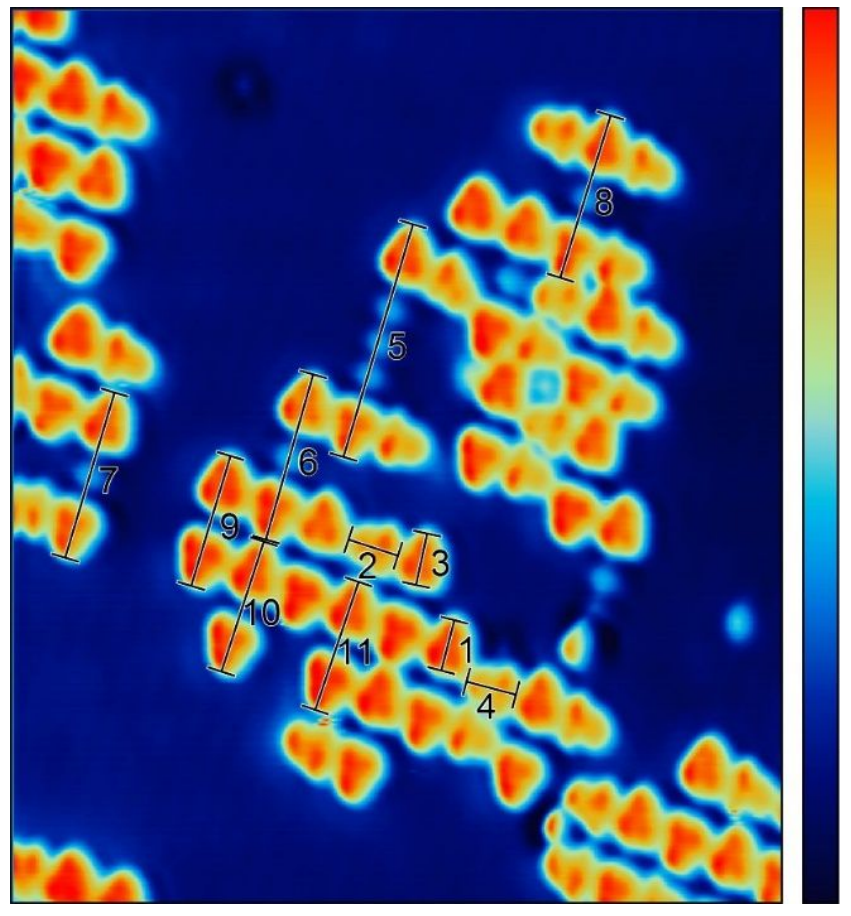

\begin{tabular}{|c|c|c|c|c|}
\hline $\begin{array}{c}\text { Length } \\
(\mathrm{nm})\end{array}$ & Single species & $\begin{array}{c}\text { Back-to-back } \\
\text { molecules }\end{array}$ & $\begin{array}{c}\text { Two molecules with } \\
\text { one bright spot }\end{array}$ & $\begin{array}{c}\text { Two molecules with } \\
\text { three bright spots }\end{array}$ \\
\hline Count 1 & 0.74 (Line 1) & 1.94 (Line 9) & 2.45 (Line 6) & 3.47 (Line 5) \\
\hline Count 2 & 0.74 (Line 2) & $1.95($ Line 10) & $2.46($ Line 7) & \\
\hline Count 3 & $0.75($ Line 3) & $1.93($ Line 11) & 2.43 (Line 8) & \\
\hline Count 4 & 0.73 (Line 4) & & & 3.47 \\
\hline Average & 0.74 & 1.94 & 2.45 & \\
\hline
\end{tabular}

Figure S3: Supplementary size information for the 1,2-BDT-Cu complexes in Fig. 1b. 
(a)

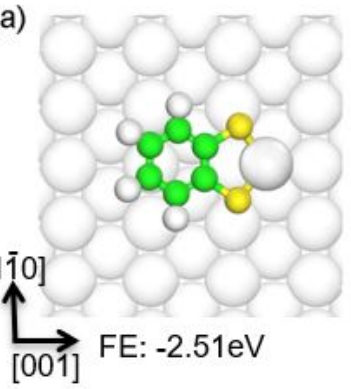

(b)

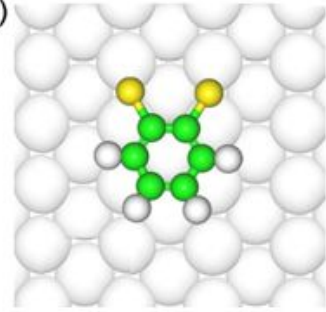

FE: $-2.21 \mathrm{eV}$ (c)

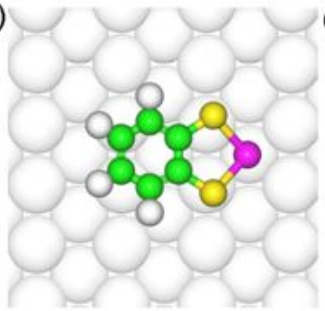

FE: $-3.10 \mathrm{eV}$ (d)

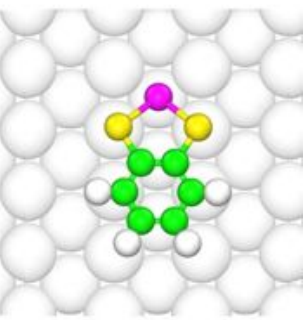

FE: $-3.34 \mathrm{eV}$ (e)

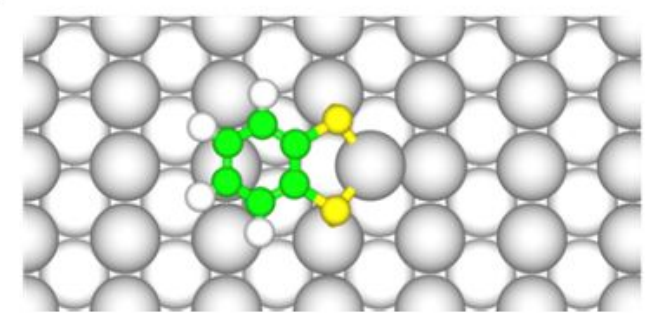

(f)

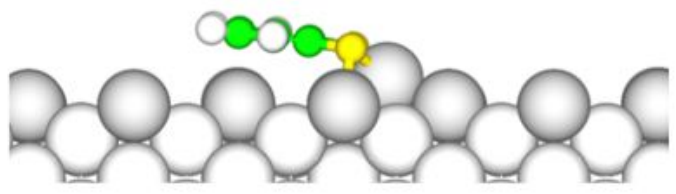

Figure S4: Four ab initio atomic structures among all 16 considered candidates for adsorbed 1,2BDT molecules on $\mathrm{Cu}(110)$. In panels (a) and (b), 1,2-BDT complex adsorb on the surface directly with formation energies (FEs) of -2.51 and $-4.21 \mathrm{eV}$, respectively. In panels (c) and (d), 1,2-BDT with a $\mathrm{Cu}$ adatom complex adsorb on $\mathrm{Cu}(110)$ with FEs of -3.10 and $-3.34 \mathrm{eV}$, respectively. The configuration with one 1,2-BDT molecule coordinated with a $\mathrm{Cu}$ adatom is energetically more stable than that of barely adsorbed 1,2-BDT. Furthermore, the configuration with the complex oriented along the [1 $1 \overline{1} 0]$ direction has the lowest $\mathrm{FE}$ of $-3.34 \mathrm{eV}$, indicating that it is the most stable configuration. The corresponding STM simulation of (d) is also consistent with the STM images. The filled green, white, yellow, magenta, and light gray balls represent $\mathrm{C}, \mathrm{H}, \mathrm{S}, \mathrm{Cu}$ adatoms, and $\mathrm{Cu}$ surface, respectively. In (a), the $\mathrm{Cu}$ atom bonding with the two $\mathrm{S}$ atoms is not the surface adatom (different from (c) and (d)) but the shifted substrate atom (from the row to the trough), which is clearly displayed in (e) and (f). 

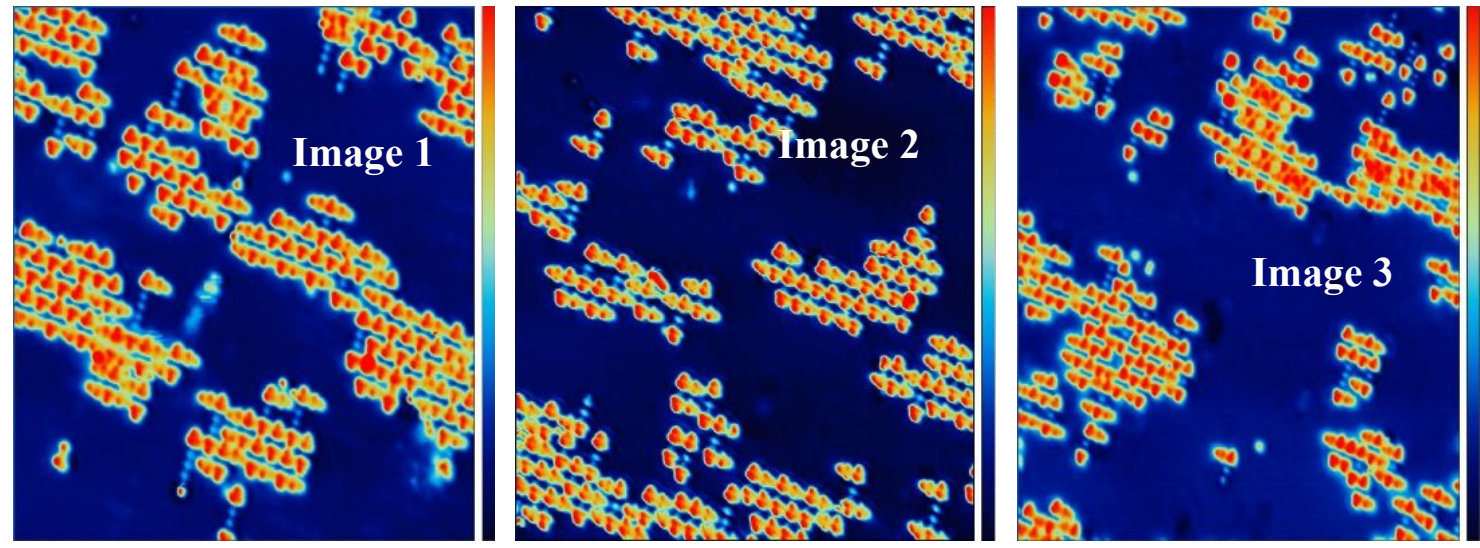

\begin{tabular}{|c|c|c|c|}
\hline Quantity of & $\begin{array}{c}1,2-\mathrm{BDT}-\mathrm{Cu} \\
\text { complexes }\end{array}$ & $\begin{array}{c}\mathrm{C} 2 \text { species in } \mathrm{Cu}-\mathrm{C} 2-\mathrm{Cu} \\
\text { chain }\end{array}$ & $\begin{array}{c}\text { C2 between two 1,2-BDT- } \\
\mathrm{Cu} \text { complexes }\end{array}$ \\
\hline Image 1 & 256 & 54 & 65 \\
\hline Image 2 & 215 & 51 & 53 \\
\hline Image 3 & 310 & 89 & $367(\mathrm{C} 2$ in total $)$ \\
\hline Sum & 781 & \multicolumn{2}{|c|}{0.47} \\
\hline Ratio & 1.00 & \multicolumn{2}{|c|}{} \\
\hline
\end{tabular}

Figure S5: Statistical analysis of the ratio between 1,2-BDT-Cu complexes and $\mathrm{C}_{2}$ fragments. Three images are used in the analysis. Image $1(-1.0 \mathrm{~V}, 100 \mathrm{pA}, 21.9 \mathrm{~nm} \times 25.5 \mathrm{~nm})$; Image $2(-$ $0.1 \mathrm{~V}, 100 \mathrm{pA}, 21.9 \mathrm{~nm} \times 25.5 \mathrm{~nm})$; Image $3(-0.1 \mathrm{~V}, 100 \mathrm{pA}, 27.4 \mathrm{~nm} \times 31.9 \mathrm{~nm})$. 


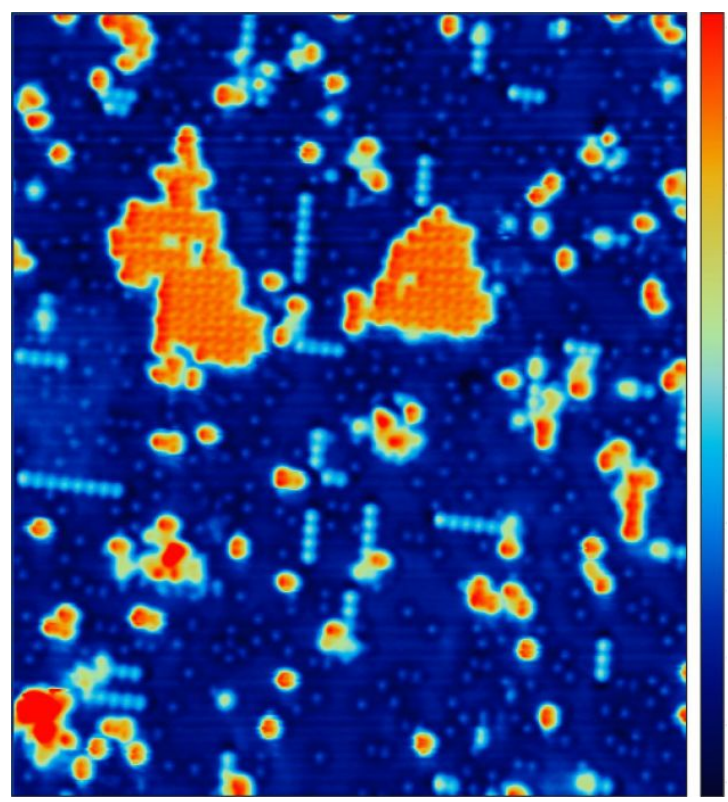

\begin{tabular}{|c|c|c|c|}
\hline Quantity of & Two lobed species & Cu-C2-Cu chain & Sulfur atoms \\
\hline Image 1 & 189 & 71 & 399 \\
\hline Image 2 & 1.00 & 0.38 & 2.11 \\
\hline
\end{tabular}

Figure S6: Statistical analysis of the chemicals species on $\mathrm{Cu}(100)$ after deposition of DBTTF molecules $(-1.0 \mathrm{~V}, 20 \mathrm{pA}, 27.1 \mathrm{~nm} \times 31.6 \mathrm{~nm})$.

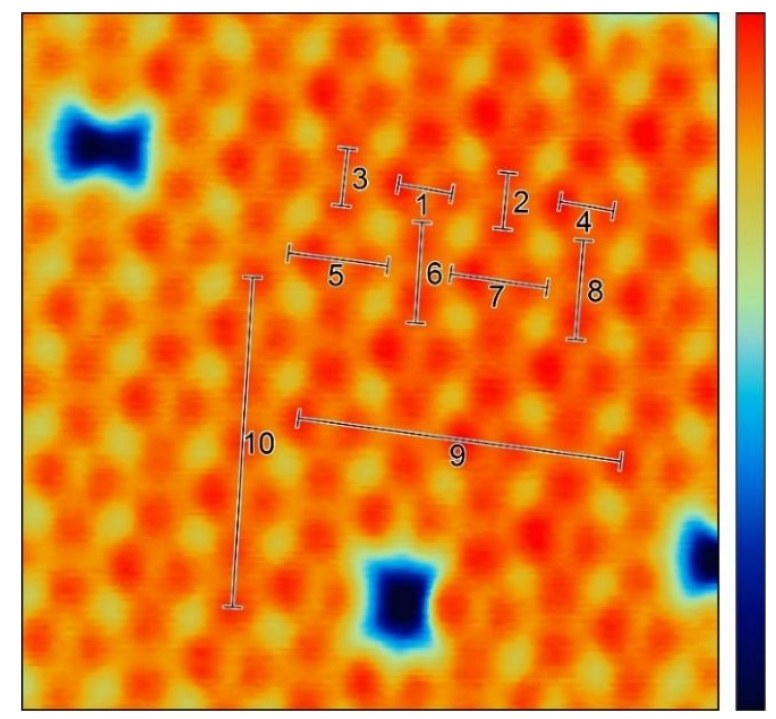

\begin{tabular}{|c|c|c|c|}
\hline Length $(\mathrm{nm})$ & Two lobes & Two adjacent molecules & Two equivalent molecules \\
\hline Count 1 & $0.35($ Line 1$)$ & $0.65($ Line 5$)$ & $1.06 * 2$ (Line 9) \\
\hline Count 2 & $0.36($ Line 2$)$ & $0.65($ Line 6$)$ & $1.07 * 2$ (Line 10) \\
\hline Count 3 & 0.37 (Line 3) & 0.63 (Line 7) & \\
\hline Count 4 & $0.35($ Line 4$)$ & $0.65($ Line 8$)$ & 1.07 \\
\hline Average & 0.36 & 0.65 & \\
\hline
\end{tabular}

Figure S7: Supplementary size information for the 1,2-phenyl diradical on $\mathrm{Cu}(100)$ in Fig. 3. 

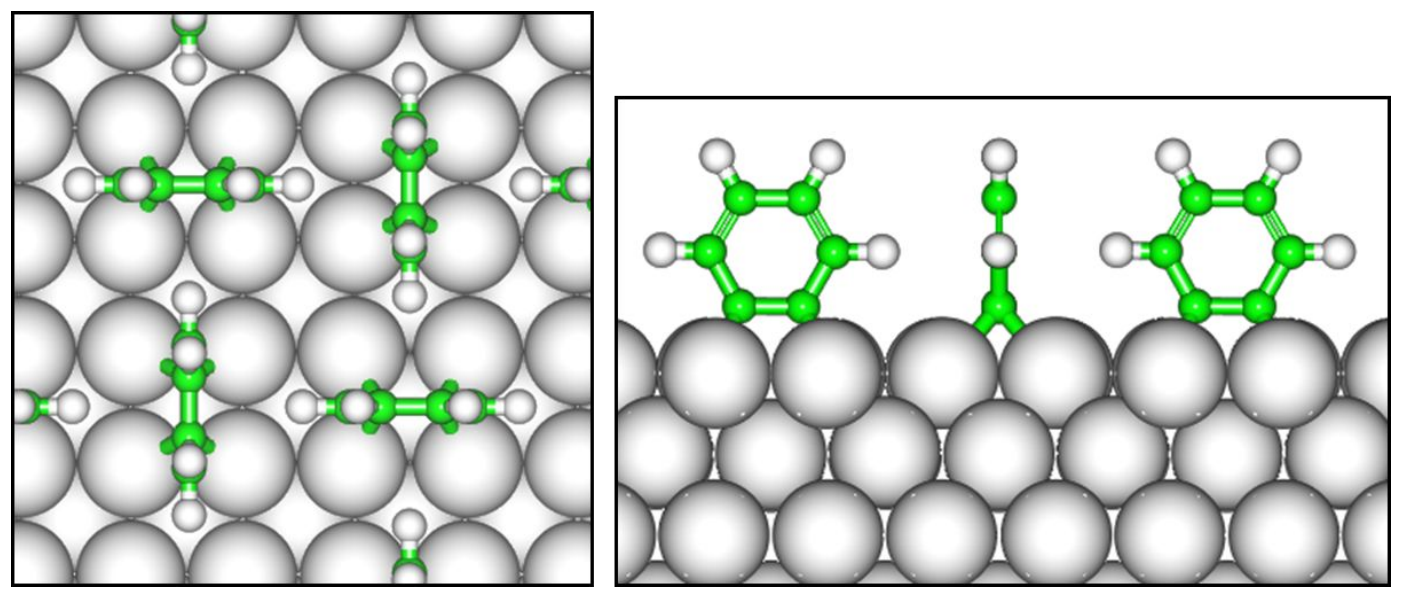

Figure S8: Top view (left) and side view (right) of the atomic structure of 1,2-phenyl diradicals on a $\mathrm{Cu}(100)$ substrate. Phenyl diradicals "stand up" via the two carbon radicals bonding to copper atoms at the bridge sites.
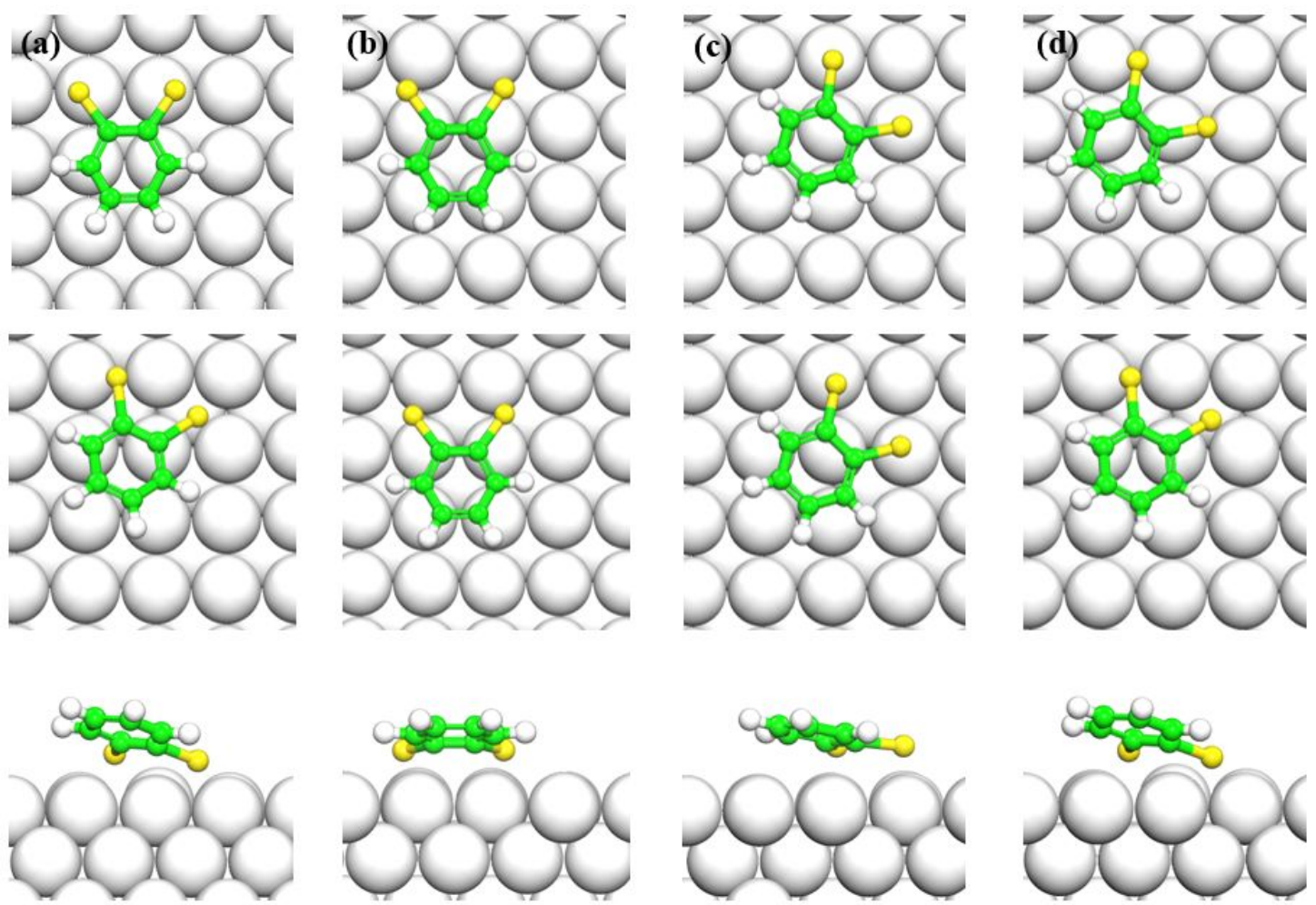

Figure S9: Atomic structure of absorbed 1,2-BDT on $\mathrm{Cu}(100)$ surface. Each column represents a possible configuration. The first two rows represent the structure before and after relaxation, and the third row shows the side view. 1,2-BDT tends to "lie down" on the surface thus it's unlikely that it would show the two-lobed feature as observed. 


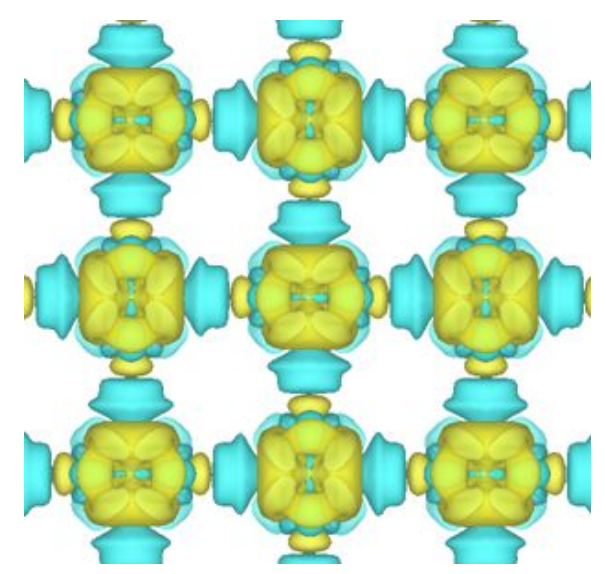

Figure S10: DCD image of the assembly structure shown in Fig. 3b-e. Intermolecular parts explicitly indicate an appreciable pi-pi stacking interaction of weak Lewis acid $\mathrm{Ph}-\mathrm{H}+$ and electronrich phenyl rings, lowering the total energy by $0.24 \mathrm{eV}$ per molecule according to our DFT calculation. This interaction primarily occurs in the middle region of the vertically adsorbed radical, which may lower the tunneling barrier width at certain positions but cannot significantly affect the charge distribution around the two topmost $\mathrm{H}$ atoms. Therefore, such intermolecular interactions cannot be clearly reflected in an STM simulation based on the Tersoff-Hamann approximation. In addition, as the tip passes through a molecule during scanning, the molecule may tilt toward the tip due to attractive interactions between them, which may result in apparent intermolecular features.
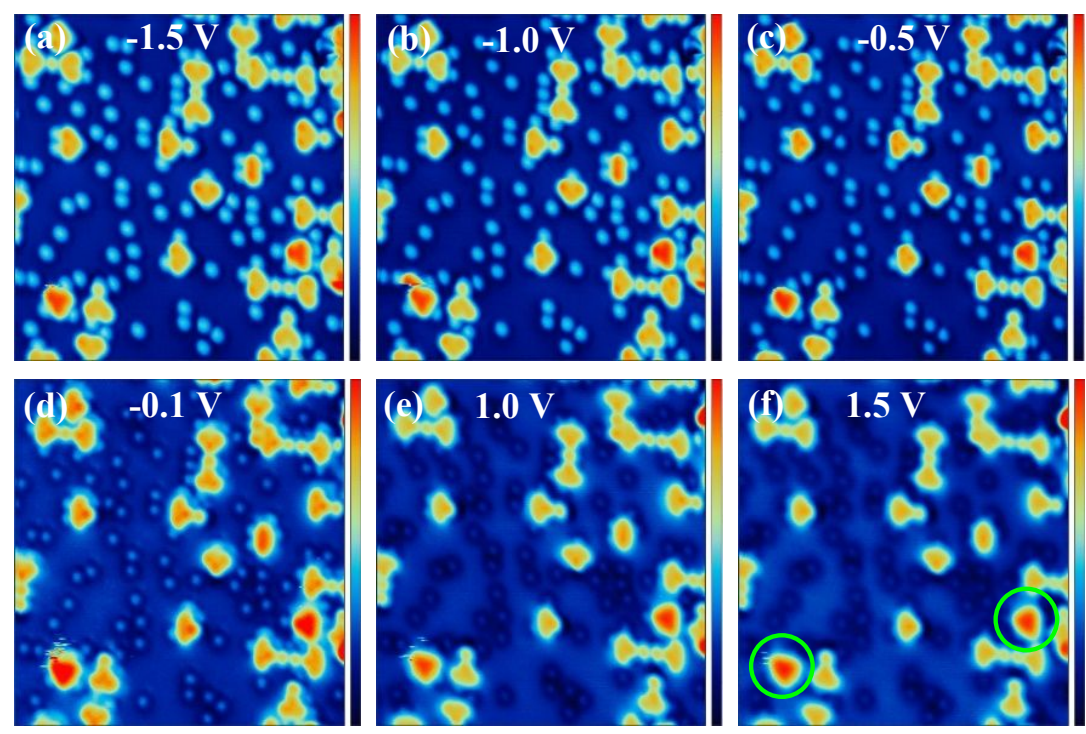

Triphenylene

Figure S11: Molecular species on $\mathrm{Cu}(100)$ after annealing at HT. STM image of chemical products on $\mathrm{Cu}(100)$ under different sample voltages $(30 \mathrm{pA}, 13 \mathrm{~nm} \times 13 \mathrm{~nm})$. The two triphenylene molecules are highlighted by green ellipses. 


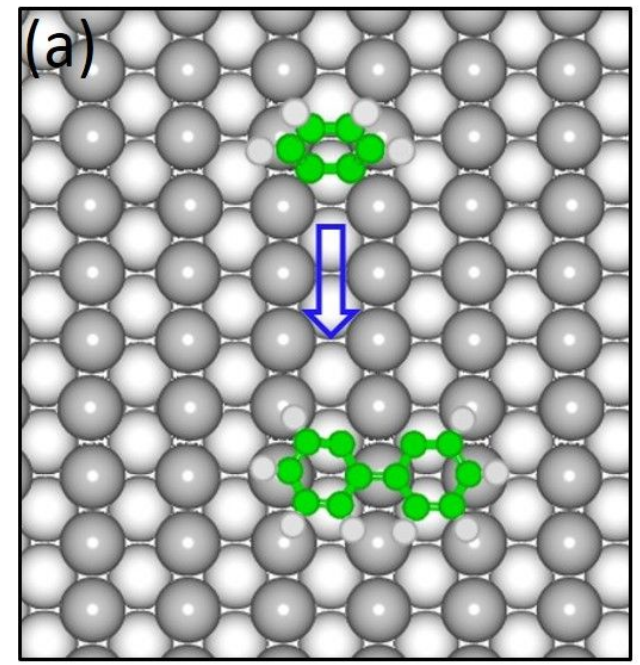

$\mathrm{Cu}(110)$

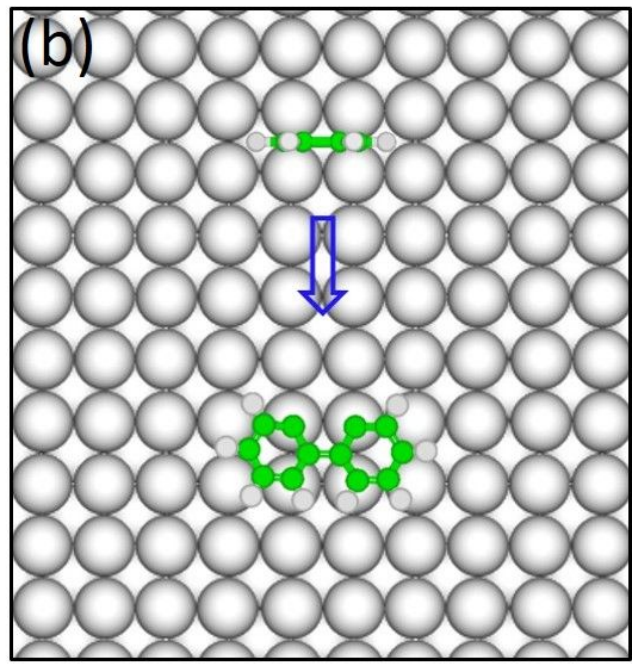

$\mathrm{Cu}(100)$

Figure S12: Schematics of the supposed facet-dependent synthesis pathways on $\mathrm{Cu}(110)$ and $\mathrm{Cu}(100)$ surfaces: (a) The phenyl diradicals can only diffuse within the troughs (shown by the arrow) on the anisotropic $\mathrm{Cu}(110)$ surface due to their preferred adsorption sites. The reaction sites of the biphenyl diradicals (from the biphenyl-Cu complex) are on the top atom row, so the phenyl diradicals almost cannot react with the biphenyl diradicals to form triphenylene. The biphenyl diradicals (from the biphenyl-Cu complex) have a few possibilities to form biphenylene by closing the four-membered ring (even though it is not very stable). (b) The phenyl diradicals can diffuse along the $<110>$ direction (shown by the arrow) on the flat $\mathrm{Cu}(100)$ surface. The reaction sites of the biphenyl diradicals (from the biphenyl-Cu complex) are only along the diffusion path of the phenyl diradicals, so they can react with each other to form triphenylene. 